\title{
Structure of Giant Nuclear Molecules
}

\author{
P. O. Hess \\ Department of Physics and Applied Mathematics, University of Cape Town, Rondebosch 7700, Cape, Republic of South Africa, \\ and Gesellschaft für Schwerionenforschung, D-6100 Darmstadt 11, West Germany \\ and \\ W. Greiner \\ Institut fur Theoretische Physik, J. W. Goethe-Universität Frankfurt, D-6000 Frankfurt am Main, West Germany \\ and \\ W. T. Pinkston \\ Department of Physics and Astronomy, Vanderbilt University, Nashville, Tennessee 37235
}

(Received 27 December 1983)

\begin{abstract}
Strong indirect evidence exists for the existence of attractive forces between nuclei making surface contact. Experimentally, the recent observations of spontaneous positron production in heavy-ion collisions can only be understood if nuclei stick together for times long compared to the collision time. We show that any such tendency for nuclei to attract implies the existence of nuclear molecules with entirely new kinds of collective modes. We present a simple model for these modes and apply it to ${ }^{238} \mathrm{U}-{ }^{238} \mathrm{U}$
\end{abstract}

PACS numbers: $25.70 . \mathrm{Jj}, 21.30 .+\mathrm{y}$

There seems to be little doubt that spontaneous positrons have been observed in recent $1^{1,2}$ heavy-ion experiments. The theoretical analysis ${ }^{1-3}$ of the data requires the nuclei to stick together for times $T$ of order $10^{-19} \mathrm{sec}$. Because the experiments are done at nominally sub-Coulomb energies, it was initially difficult to understand how the nuclei could get sufficiently close together for attractive nuclear forces to act. However, double-folding-model calculations ${ }^{4}$ have shown that the nuclear plus Coulomb interactions of strongly deformed nuclei show a dramatic dependence on nuclear orientation. These calculations predict the formation of potential barriers which are lower by $100 \mathrm{MeV}$ when the nuclei approach with their symmetry axes collinear than in the least favorable case, in which they approach so that their equators touch. Although the foldingmodel predictions cannot be relied upon at shorter distances where there is significant overlap of nuclear densities, they suggest the very real possibility that a potential energy minimum exists in the nuclear surface-a "pocket," depending on orientation, which can capture the nuclei. Improved potential models are under study, and preliminary calculations ${ }^{5}$ suggest the existence of pockets. The purpose of this Letter is to show that pockets of only a few megaelectronvolts in depth are needed to produce a rich spectroscopy of quasimolecular resonances, and to present a simple model for the structure of some of these states of heavy nuclear molecules.
Quantum mechanically capture behind a barrier and the resultant delay time imply a resonance, of width related to delay time $T$ according to

$$
\Gamma \sim \hbar / T \text {. }
$$

The "sticking times" required correspond to $50-100 \mathrm{keV}$. A simple estimate can be made of the minimum depth $D$ needed to produce a resonance this width. Consider the potential $V$ sketched in Fig. 1(a). A resonance is expected at the energy $\frac{1}{2} \hbar \omega$ above the bottom of the pocket. The width of this resonance can be estimated from the HillWheeler penetrability formula ${ }^{6}$ :

$$
\Gamma=\frac{\hbar \omega}{2 \pi}\left\{1+\exp \left[\frac{2 \pi\left(D-\frac{1}{2} \hbar \omega\right)}{\hbar \omega}\right]\right\}^{-1} .
$$

In Eq. (1) we have assumed that the curvature $(\hbar \omega)$ of the barrier top is the same as that of the minimum. It can be estimated in the following way, from essentially dimensional considerations: Let the Coulomb potential, which varies slowly compared to the nuclear potential, be approximated in the region of contact by a linear function. Let the nuclear contribution fall off exponentially, as $\exp (-r / a)$. Then, at the potential maximum, we have

$$
\frac{d^{2} V}{d r^{2}}=\frac{-1}{a} \frac{d V_{\text {Coul }}}{d r}
$$

For the U-U system, the Coulomb repulsion 

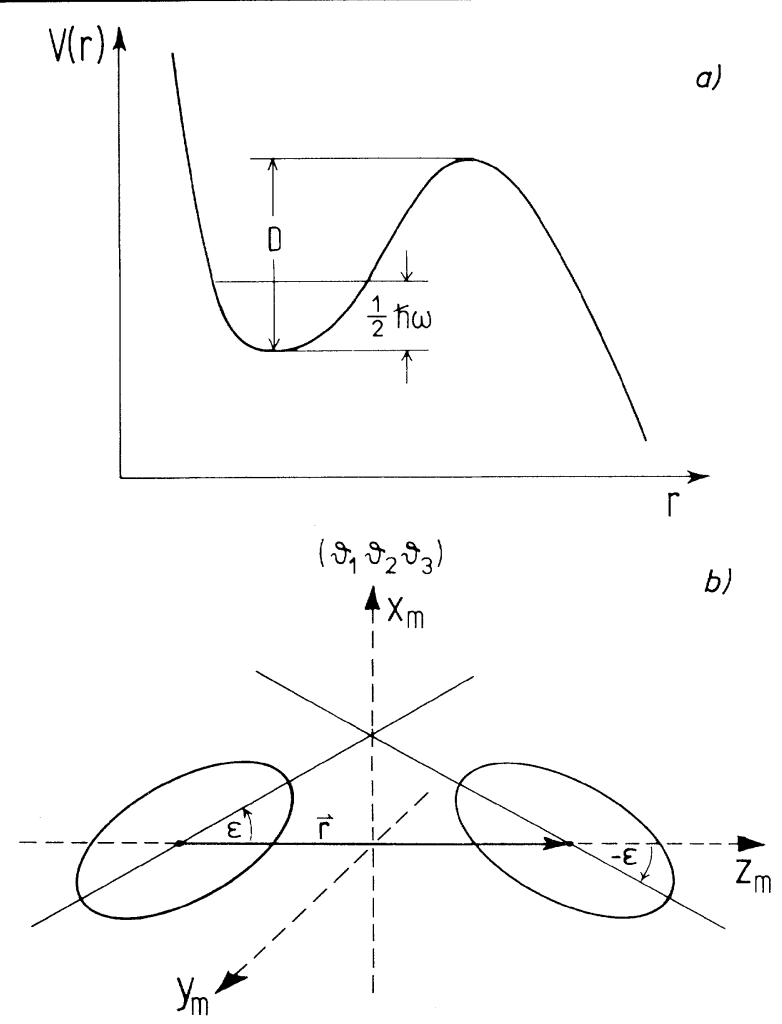

FIG. 1. (a) Sketch of potential with an attractive pocket, showing the location of the lowest energy resonance. (b) Simple model for the "butterfly" and "belly dancer" modes, showing the symmetry and the principal axes of rotation.

changes by $30-40 \mathrm{MeV}$ per femtometer in the surface region. $a$ is of the order $1.0 \mathrm{fm}$, so that the "spring constant" is of the order $30 \mathrm{MeV} / \mathrm{fm}^{2}$, and $h \omega$ is about $3 \mathrm{MeV}$. Assuming a width of $100 \mathrm{keV}$ results in $D \approx 2 \mathrm{MeV}$. These estimates agree well with the curvature of the double-folding barrier. Another feature of these molecular resonances is that they should exist with rather large values of angular momentum. Because of the large moment of inertia of these systems, the depths of the pockets in the effective potential energy (potential energy plus centrifugal stretching) vary slowly with angular momentum $I$; the pockets persist up to values of $I=100-200$, or more. A pocket several megaelectronvolts deep will result in one or more rotational bands of hundreds of levels. These bands will cover an energy of 10-20 MeV. This is an important consideration in connection with the positrons. Because of straggling in the target, the effective beam energy has spread of order $10 \mathrm{MeV}$. As a result an isolated resonance of width $\sim 100 \mathrm{keV}$ would be unlikely to have an observable effect in such an experiment. The molecular model does not suffer (a)

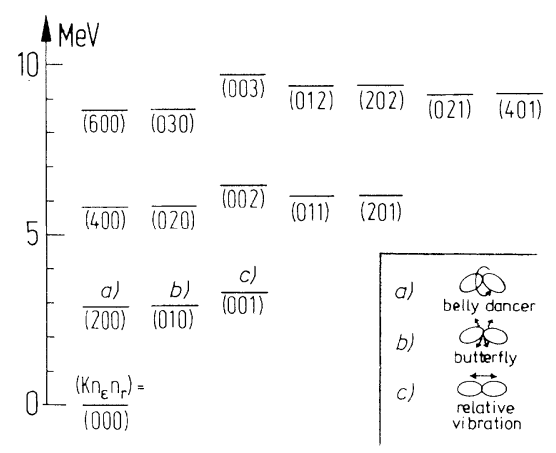

(b)

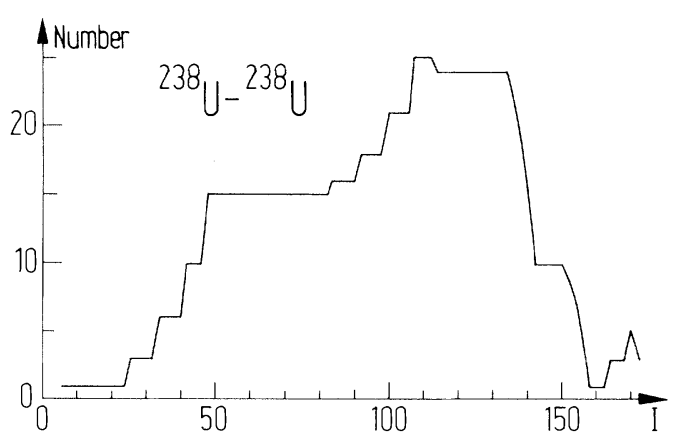

FIG. 2. (a) Energy level diagram for the vibrational states of the giant nuclear molecule ${ }^{238} \mathrm{U}_{-}{ }^{238} \mathrm{U}$ and (b) distribution of angular momentum states in a $10 \mathrm{-MeV}$ window.

from this difficulty; it predicts many resonances, spread over several megaelectronvolts. The calculated level density, if supplied by the excitation of individual $\beta-\gamma$ vibrations of the two nuclei, yields the order of magnitude of the observed cross section. Moreover, recent calculations ${ }^{7}$ of the influence of such resonances on the positron production show that the band structure plays an essential role in the positron production theory.

This oversimplified treatment is essentially one dimensional; it treats vibrational states in the relative motion. There are also quantized motions in the orientation variables. These motions correspond physically to hindered rotations of the deformed nuclei, each moving in the quadrupole field produced by the other. Two of these new kinds of collective vibrations are pictured, along with the vibration in the $r$ coordinate, in Fig. 2(a). We call these "butterfly" and "belly dancer" modes of vibrations. Some of the new molecular collective modes can also play a role in the fission of nuclei. ${ }^{8}$ A simple collective model for these motions is given below.

Before we proceed, it is instructive to compare 
the cohesive mechanism discussed here with that of the dual resonance ${ }^{9}$ of quasi-molecular states in light nuclei. In collisions of ${ }^{16} \mathrm{O}$ on ${ }^{16} \mathrm{O}$, for example, internal excitations of the colliding, spherical nuclei reduce their relative kinetic energy, so that they drop behind the barrier and are trapped. Completely analogous would be the situation in which $\beta$ and $\gamma$ vibrations of the $U$ nuclei were excited. These have so far been neglected in our treatment; however, excitations of internal states play a key role here also. What is different in the present model is the strong dependence of $V$ on the orientation. Favorable orientations are achieved by coherent excitation of rotational states of the individual nuclei. Our description, in terms of rotations of the system as a whole, superimposed on coupled, hindered rotations of the individual nuclei, is equivalent to, but more effective and physically appealing than, the description in terms of the mutual excitation of rotational states.

We consider a system of identical nuclei such as ${ }^{238} \mathrm{U}-{ }^{238} \mathrm{U}$, which are assumed to be axially symmetric. They are constrained as shown in Fig. 1(b) so that both symmetry axes lie in a common plane with the relative vector $\vec{r}$. Also we require their orientation angles to be equal and opposite. $\epsilon_{1}=-\epsilon_{2}=\epsilon$. The butterfly mode is an oscillation in the variable $\epsilon$. The belly-dancer mode is a rotation of the plane containing the symmetry axes.
For a very deep pocket these constraints are very reasonable. Even when the pocket is not so deep this model gives a good qualitative picture of some of the motions.

The classical kinetic energy for the physical system in Fig. 1(b) is given by

$$
T=\frac{1}{2} \Sigma_{k} \theta_{k k} \omega_{k}^{\prime 2}+3 B \beta_{0}^{2} \dot{\epsilon}^{2}+\frac{1}{2} \mu \dot{r}^{2} .
$$

The $\omega_{k}^{\prime}$ are angular velocities measured in the intrinsic frame, $\beta_{0}$ is the deformation of the nuclear ground state, and $B$ is the collective inertia parameter. The notation is that of Eisenberg and Greiner. ${ }^{10}$ The reduced mass of the sytem is $\mu$. The term proportional to $\dot{\epsilon}^{2}$ corresponds to the butterfly motion. The belly-dancer motion is a rotation about the $z_{m}$ axis. The principal axes and moments of inertia of the system of Fig. 1(b) are easily determined. The moments are

$$
\begin{aligned}
& \theta_{11}=6 B \beta_{0}^{2} \cos ^{2} \epsilon+\mu r^{2}, \quad \theta_{22}=6 B \beta_{0}^{2}+\mu r^{2}, \\
& \theta_{33}=6 B \beta_{0}^{2} \sin ^{2} \epsilon .
\end{aligned}
$$

For the potential energy we choose

$$
V=\frac{1}{2} C_{\epsilon} \epsilon^{2}+\frac{1}{2} C_{r}\left(r-r_{0}\right)^{2} .
$$

Pauli quantization of this system is carried out as described in Ref. 10 including a change of volume element. When $\hat{L}_{k}^{\prime}(k=1,2,3)$ are the components of the angular momentum operator in the molecular frame, we obtain for the Hamiltonian

$$
\hat{H}=\frac{\hbar^{2}}{2} \sum_{k} \frac{\hat{L}_{k}^{\prime 2}}{2 \theta_{k \kappa}}-\frac{\hbar^{2}}{12 B \beta_{0}^{2}} \frac{\partial^{2}}{\partial \epsilon^{2}}-\frac{\hbar^{2}}{2 \mu} \frac{\partial^{2}}{\partial r^{2}}+\frac{1}{2} C_{\epsilon} \epsilon^{2}+\frac{1}{2} C_{r}\left(r-r_{0}\right)^{2}-\frac{\hbar^{2}\left(1+\sin ^{2} \epsilon\right)}{48 B \beta_{0}^{2} \sin ^{2} \epsilon} .
$$

We assume small vibrations and approximate $\hat{H}$ by expanding in powers of $\epsilon$ and $\bar{r}=r-r_{0}$. The lowest-order Hamiltonian is

$$
\hat{H}_{0}=\frac{\hbar^{2}}{2 \theta_{0}}\left(\hat{L}^{2}-\hat{L}_{3}^{\prime 2}\right)+\frac{\hbar^{2} \hat{L}_{3}^{\prime 2}}{12 B \beta_{0}^{2} \epsilon^{2}}-\frac{\hbar^{2}}{12 B \beta_{0}^{2}} \frac{\partial^{2}}{\partial \epsilon^{2}}-\frac{\hbar^{2}}{48 B \beta_{0}^{2} \epsilon^{2}}+\frac{1}{2} C_{\epsilon} \epsilon^{2}-\frac{\hbar^{2}}{2 \mu} \frac{\partial^{2}}{\partial \bar{r}^{2}}+\frac{1}{2} C_{r} \bar{r}^{2},
$$

with

$$
\theta_{0}=6 B \beta_{0}^{2}+\mu r_{0}^{2} \text {. }
$$

Equation (4) has the same mathematical structure as the Hamiltonian of the rotation-vibration model (RVM).$^{10,11}$ Its eigensolutions are similar to those of the RVM with one difference: The projection of the angular momentum on the molecular $z$ axis $K$ is replaced by $2 K$, because of different factors of the terms $\sim \hat{L}_{0}^{\prime 2}$ and $\sim \epsilon^{-2}$. The wave functions must be symmetrized ${ }^{10}$ so that they are single-valued functions of the laboratory coordinates. The result is

$$
\left|I \mu K n_{\epsilon} n_{r}\right\rangle=\left[\frac{2 I+1}{16 \pi^{2}\left(1+\delta_{k 0}\right)}\right]^{1 / 2}\left[D_{\mu K}^{I^{*}}+(-1)^{I} D_{\mu-K}^{I^{*}}\right] \chi_{2 K, n_{\epsilon}}(\epsilon) g_{n_{r}}(\bar{r}) .
$$

The function $g$ is a one-dimensional harmonic oscillator function, and $\chi$ is given in Ref. 10. The energy is given in Ref. 10. The energy is given by

$$
E=\frac{\hbar^{2}}{2 \theta_{0}}\left[I(I+1)-K^{2}\right]+\hbar^{2}\left(\frac{C_{r}}{\mu}\right)^{1 / 2}\left(n_{r}+\frac{1}{2}\right)+\hbar\left(\frac{C_{\epsilon}}{6 B \beta_{0}^{2}}\right)^{1 / 2}\left(|K|+2 n_{\epsilon}+1\right)
$$


with $K=0,2,4, \ldots ; I=0,2,4, \ldots, \quad$ if $K=0$, and $I=K, K+1, K+2, \ldots$, if $K \neq 0$. Because a system of identical nuclei is treated, the wave function must be symmetric under $\epsilon \rightarrow-\epsilon$. Because of this symmetry, $K$ must be even. The structure of the energy eigenvalue formula shows that the $r$ and $\epsilon$ motions of the giant nuclear molecule are analogous to the $\beta$ and $\gamma$ motions of ordinary deformed nuclei.

A calculated spectrum for the U-U system is shown in Fig. 2(a). The parameters $C_{\epsilon}$ and $C_{r}$ were taken from Ref. 5, and the others from RVM treatments of ${ }^{238} \mathrm{U}$. This potential has fairly deep pockets, $20 \mathrm{MeV}$ deep for the most favorable orientation. The parameters are $\hbar^{2} / 2 \theta_{0}=0.57 \times 10^{-3} \mathrm{MeV}$, $C_{r}=30 \mathrm{MeV} / \mathrm{fm}^{2}$, and $C_{\epsilon}=279 \mathrm{MeV} / \mathrm{rad}^{2}$. Only bandheads are shown in Fig. 2(a). For each band there is a rotational band of closely spaced levels. Each band is classified by the quantum numbers $\left(K, n_{\epsilon}, n_{r}\right)$. For example, $(2,0,0) \quad I=2$ and $(0,1,0)$ $I=0$ are pure belly-dancer and butterfly modes, respectively. In order to illustrate the number of resonant states in a given energy range, we show in Fig. 2(b) the distribution in angular momentum of energy levels in an energy window of $10 \mathrm{MeV}$. The energy window is centered near the top of the barrier for $I=0$. Not all states in this window are included, only those which would make a nonnegligible contribution ${ }^{12}$ to the positron yield $(1 \mathrm{keV}$ $<\Gamma<10 \mathrm{MeV})$. The widths were estimated using the Hill-Wheeler formula. The total number of states represented in Fig. 2(b) is of order 1000. The angular momentum distribution is peaked about an average value of $(100-120) \hbar$.

Not all of the collective modes of giant nuclear molecules have been treated here. In reality nuclei are not constrained to move so that their symmetry axes are in the same plane. Relaxing this constraint means that there need not be symmetry under $\epsilon \rightarrow-\epsilon$, and $K$ need not always be even. Numerical calculations have been made ${ }^{13}$ which are free of these constraints, and they indicate that the odd- $K$ bands have excitation energies of the same order as the even- $K$ bands.
Clearly the existence of cohesive forces between the surfaces of deformed nuclei leads to a rich variety of entirely new collective modes. These quasimolecular states have properties needed to explain the spontaneous positrons observed in heavyion collisions. Their existence would influence other processes; for example, they may be "doorways" for sub-barrier fusion.

We gratefully acknowledge many helpful discussions with J. A. Maruhn, M. Seiwert, and U. Heinz.

${ }^{1}$ M. Clemente, E. Berdemann, P. Kienle, H. Tsertos, W. Wagner, F. Bosch, C. Kozhuharov, and W. Koenig, in Proceedings of the International Conference on Nuclear Physics, Florence, 29 August-3 September 1983, edited by R. Ricci and P. Blasi (Tipografia Compositori, Bologna, (1984), p. 693.

${ }^{2}$ H. Bokemeyer, H. Folger, H. Grein, T. Cowan, J. S. Greenberg, J. Schweppe, K. Bethge, A. Gruppe, K. E. Stiebing, D. Schwalm, P. Vincent, and N. Trautmann, in Proceedings of the International Conference on Nuclear Physics, Florence, 29 August-3 September 1983, edited by R. Ricci and P. Blasi (Tipografia Compositori, Bologna, 1984), p. 694.

3J. Reinhardt, U. Müller, B. Müller, and W. Greiner, Z. Phys. A 303, 173 (1981).

${ }^{4}$ M. J. Rhoades-Brown, V. E. Oberacker, M. Seiwert, and W. Greiner, Z. Phys. A 310, 287 (1983).

${ }^{5} \mathrm{M}$. Seiwert, unpublished.

${ }^{6}$ D. L. Hill and J. A. Wheeler, Phys. Rev. 89, 1102 (1953).

${ }^{7}$ U. Heinz, J. Reinhardt, B. Müller, W. Greiner, and W. T. Pinkston, to be published.

${ }^{8}$ J. R. Nix and W. Swiatecki, Nucl. Phys. 71, 1 (1965).

${ }^{9}$ W. Scheid, W. Greiner, and R. Lemmer, Phys. Rev. Lett. 25, 176 (1970).

${ }^{10} \mathrm{~J}$. M. Eisenberg and W. Greiner, Nuclear Theory $I$ (North-Holland, Amsterdam, 1975).

${ }^{11}$ A. Faessler and W. Greiner, Z. Phys. 168, 425 (1962); A. Faessler and W. Greiner, Z. Phys. 170, 105 (1962); A. Faessler and W. Greiner, Z. Phys. 177, 190 (1964); A. Faessler, W. Greiner, and R. K. Sheline, Nucl. Phys. 80, 417 (1965).

${ }^{12}$ U. Heinz, J. Reinhardt, B. Müller, W. Greiner, and U. Müller, Z. Phys. A 314, 125 (1983).

${ }^{13} \mathrm{~W}$. T. Pinkston, unpublished. 